$11 \mid 1989$

Varia

\title{
Pascal en Amérique
}

(1980-1989)

\section{Pierre Force}

\section{(2) OpenEdition Journals}

Édition électronique

URL : http://journals.openedition.org/ccibp/645

DOI : $10.4000 /$ ccibp. 645

ISSN : 2493-7460

Éditeur

Centre international Blaise Pascal

\section{Édition imprimée}

Date de publication : 20 décembre 1989

Pagination : 25-27

ISSN : 0249-6674

Référence électronique

Pierre Force, «Pascal en Amérique », Courrier du Centre international Blaise Pascal [En ligne], 11 | 1989, mis en ligne le 11 décembre 2015, consulté le 08 mai 2019. URL : http://journals.openedition.org/ ccibp/645; DOI : 10.4000/ccibp.645

Ce document a été généré automatiquement le 8 mai 2019.

Centre international Blaise Pascal 


\title{
Pascal en Amérique
}

(1980-1989)

\author{
Pierre Force
}

Quoique Pascal ne soit pas un des auteurs les plus étudiés en Amérique du Nord, l'immensité du continent et le nombre des universités américaines et canadiennes expliquent la relative abondance des articles, thèses et publications consacrés à Pascal depuis 1980. Dans les principales universités des États-Unis, neuf « dissertations » (thèses de doctorat) sur Pascal ont été soutenues entre 1980 et 1988 (ce chiffre ne comprend pas les thèses de littérature comparée, genre très florissant aux États-Unis en ce moment). De 1980 à 1989, treize livres ayant pour sujet l'œuvre de Pascal ont été publiés par des universitaires travaillant en Amérique du Nord. Le plus récent est une monographie composée d'articles pour la plupart classiques, préfacée par Harold Bloom, qui est sans doute le plus célèbre et le plus respecté des critiques littéraires américains d'aujourd'hui.

Qui sont les pascalisants américains? On n'insistera pas sur les "anciens", que les spécialistes de Pascal connaissent bien: Jean-Jacques Demorest, qui publia dans les années 50 de très belles études sur le style de Pascal et a édité en 1984 les actes du colloque Pascal et Corneille de Tucson (Arizona) ; Hugh Davidson ${ }^{1}$ à qui l'on doit, outre les indispensables concordances des Pensées et des Provinciales, un livre rigoureux sur le sens apologétique des Pensées, et une splendide monographie sur notre auteur ; Anthony Pugh ${ }^{2}$ , auteur d'une ambitieuse somme qui interprète et justifie le classement des liasses à titres des Pensées ; Robert Nelson ${ }^{3}$ qui, dans son étude de la conversion chez Pascal, met en évidence de façon magistrale l'interaction de l'argumentation et du vécu; Jan Miel ${ }^{4}$, auteur d'un ouvrage classique sur la théologie de Pascal ; Édouard Marot-Sir, homme aux multiples talents, qui, dans son livre remarquable sur la métaphysique de Pascal, pose et démontre la thèse selon laquelle Pascal est un philosophe dont la doctrine doit être prise au sérieux; Lane Relier ${ }^{5}$, éditeur (avec Jan Richmond) du très beau recueil d'articles récemment paru chez Vrin; et enfin, les plus jeunes des anciens, David Jaymes ${ }^{6}$, auteur d'articles à orientation philosophique, et Buford Norman ${ }^{7}$, qui a publié plusieurs articles sur la logique et la rhétorique de Pascal, et, tout récemment, un livre intitulé Portraits of Thought: Knowledge, Methods and Styles in Pascal. 
Cet article voudrait faire connaître les jeunes; sont ici qualifiés de «jeunes » ceux dont les premiers travaux consacrés à Pascal sont postérieurs à 1980. David Wetsel ${ }^{8}$ a publié en 1981 une très savante étude portant le beau titre (cité de Pascal) L'Écriture et le Reste. Analysant une œuvre longtemps ignorée des pascalisants, la Bible traduite et commentée par Le Maistre de Sacy, M. Wetsel multiplie les rapprochements avec les Pensées, et replace ainsi le projet apologétique de Pascal dans la tradition exégétique port-royaliste. Orné de nombreuses gravures tirées de la Bible de Sacy, cet ouvrage contribue, comme l'écrit Philippe Sellier dans sa préface, "à l'ouverture d'un champ ", qui est celui des rapports entre exégèse biblique et création littéraire au XVII siècle. M. Wetsel est aussi l'organisateur du mini-colloque intitulé « Meaning, Structure and History in the Pensées of Pascal » qui s'est tenu l'université d'État de Portland (Oregon) les 5 et 6 avril 1989.

Nietzsche and Pascal on Christianity (New York, 1985), de Charles Natoli ${ }^{9}$ est l'œuvre d'un croyant qui pose avec rigueur la question des rapports entre foi et raison en établissant une sorte de dialogue entre l'antichristianisme polémique de Nietzsche et la pensée de celui que Nietzsche appelait "le seul chrétien logique». Dans un renversement dialectique, M. Natoli montre que par son usage à la fois honnête et habile du doute, Pascal est paradoxalement le moins dogmatique des deux penseurs.

5 Sara Melzer ${ }^{10}$ a publié en 1986 une étude des Pensées intitulé Discourses of the Fall, qui est très lue et très commentée par les pascalisants américains. S'inspirant des travaux de Jacques Derrida et de l'interprétation que donne Lucien Goldmann de la théologie négative de Port-Royal, $\mathrm{M}^{\mathrm{me}}$ Melzer montre que pour Pascal, l'homme d'après la chute dispose d'un langage irréductiblement ambigu, qui fait sombrer dans l'aporie toute tentative de discours sur la vérité divine. Sans nier l'intention apologétique des Pensées, Sara Melzer montre que la nature même du langage humain d'après la chute rend cette intention hautement problématique. Appliquant à la lecture de Pascal lui-même la théorie de la lecture qu'elle dégage des Pensées, $\mathrm{M}^{\mathrm{me}}$ Melzer montre que la signification des Pensées est foncièrement indécidable.

6 Prenant à certains égards un parti inverse de celui de Sara Melzer, Louis MacKenzie ${ }^{11}$, auteur de Pascal's Lettres Provinciales : the Motif and Practice of Fragmentation (1988), insiste sur l'effort de cohérence et d'univocité qui se manifeste dans les Provinciales. Pour M. MacKenzie, ce qui est en jeu dans la polémique avec les jésuites est le rapport entre le tout et la partie: les "mots magiques» des jésuites sont des énoncés performatifs («speech acts») indépendants les uns des autres, qui se font accepter en dissimulant leurs conditions d'énonciation. Selon Louis MacKenzie, c'est en fragmentant à son tour et à sa façon les traités jésuites et en effectuant un «collage » de leurs maximes que Pascal met en évidence le caractère aberrant de leur doctrine. Dans la perspective qui est la sienne, M. MacKenzie conclut en définissant la vérité comme unité et absence de fragmentation.

7 Certains universitaires qui s'étaient illustrés dans d'autres domaines de recherche ont récemment consacré d'intéressants articles à Pascal. C'est le cas de Domna Stanton ${ }^{12}$ qui a publié un long article sur l'ordre et la fragmentation. Citons aussi Barbara Woshinsky ${ }^{13}$ auteur de deux articles sur la lecture pascalienne de l'Écriture.

8 Parmi les plus jeunes, Milad Doueihi ${ }^{14}$ a consacré une partie de son doctorat de littérature comparée (dont la publication est prochaine) à des analyses brillantes de l'Eucharistie chez Pascal, inspirées des travaux de Louis Marin. John Gallucci ${ }^{15}$, auteur d'un article intitulé « Pascal poeta-theologus » (à paraître dans les PFSCL), s'intéresse aux tensions non 
résolues entre poésie et théologie dans l'œuvre de Pascal, a consacré sa thèse de doctorat aux rapports entre science et rhétorique, et travaille à présent sur la théorie pascalienne des figures.

9 Considérant l'immensité et la diversité des pays qu'il envisage, ce compte-rendu est condamné à être incomplet. Le Québec, membre de la communauté francophone, et dont les publications sont mieux connues en Europe, est omis. Est omise aussi toute l'Amérique latine. Les auteurs de travaux récents effectués dans le Nouveau Monde et qui auraient été omis dans cet article sont instamment priés de se faire connaître auprès du CIBP, qui publiera les addenda et mises à jour nécessaires.

\section{BIBLIOGRAPHIE}

\section{Ouvrages publiés depuis 1980 par les chercheurs travaillant en Amérique du Nord (Québec excepté)}

BLOOM (Harold) [éd. et préf.], Blaise Pascal, Modern Critical View Series, New York, Chelsea House, 1989. [Articles de Auerbach, Demorest, Goldmann, Mesnard, Melzer, etc.]

COLEMAN (Francis), Neither Angel nor Beast : The Life and Work of Blaise Pascal, New York et Londres, Routledge \& Kegan, 1986, 227 p.

DAVIDSON (Hugh), Blaise Pascal, Boston, Twayne, 1983, 150 p.

DEMOREST (Jean-Jacques) et LEIBACHER-OUVRARD (Lise) [éd.], Pascal, Corneille. Désert, retraite, engagement, Actes du Colloque de Tucson ; Seattle, Tübingen, PFSCL, « Biblio 17 » ${ }^{\circ}$ 21, 1984, $322 \mathrm{p}$.

FORCE (Pierre), Le problème hernéneutique chez Pascal, Paris, Vrin, 1989, 297 p.

HELLER(Lane) et RICHMOND (Ian) [éd.], Pascal. Thématique des Pensées, Paris, Vrin, 1988, 146 p. [Articles de Pugh, Mesnard, Beugnot, Davidson, Heller, Miel et Sellier]

MELZER (Sara), Discourses of the Fall, Berkeley et Los Angeles, University of California Press, 1986, $171 \mathrm{p}$.

MacKENZIE (Louis), Pascal's Lettres Provinciales : The Motif and Practice of Fragmentation, Birmingham, Alabama, Summa Publications, 1988, 135 p.

NATOLI (Charles), Nietzsche and Pascal on Christianity, New York, P.Lange, 1985, 197 p. NELSON (Robert), Pascal Adversary and Advocate, Cambridge, Massachussets, Harvard University Press, 1981,286 p.

NORMAN (Buford), Portraits of Thought: Knowledge, Methods and Styles in Pascal, Columbus, Ohio, Ohio State, University Press, 1989.

PUGH (Anthony), The Composition of Pascal's Apologia, Toronto, University of Toronto Press, 1984, 633p. 
RESCHER (Nicholas), Pascal's Wager : A Study of Practical Reasoning in Philosophical Theology, Notre-Dame, Indiana, University of Notre-Dame Press, 1985, 154p.

WETZEL (David), L'Écriture et le Reste : The Pensées of Pascal in the Exegetical Tradition of PortRoyal, Colombus, Ohio, Ohio State University Press, 1981, 234 p.

\section{Articles cites dans ce compte-rendu}

DOUEIHI (Milad), « Trap's representation », Diacritics XIV, 1, 1984, pp. 66-77.

GALLUCCI (John), « Anew source for one of Pascal's Pensées [L. 755-B.258] », Romance Notes, vol. XXVIII, $n^{\circ} 1,1987$, pp. 63-69.

JAYMES (David), « Play in Pascal's Pensées », Papers on French Seventeenth Century Literature, vol. VIII, n 14, 1981, pp. 39-49.

$\mathrm{KOCH}$ (Erec), «The perspective of science : Pascal's Préface pour le traité du vide », Papers on French Seventeenth Century Literature, vol. XV, $\mathrm{n}^{\circ} 28,1988$, pp. 149-160.

NORMAN (Buford), «Chez les jésuites et chez les honnêtes gens. Methods and styles in the Provinciales ", in Actes du Colloque de Tucson, pp. 57-58.

STANTON (Domna), « Pascal's fragmentary thoughts : Dis-order and its overdetermination », Semiotica 51(1984), pp. 211-235.

WOSHINSKY (Barbara), « Biblical discourse : Reading the unreadable », L'Esprit Créateur 21, 1981, pp. 13-24.

WOSHINSKY (Barbara), « Pascal's Pensées and the discourse of the inexpressible », PFSCL, vol. VIII, $\mathrm{n}^{\circ} 14,1981$, pp. 57-65.

\section{Thèses de doctorat sur Pascal soutenues aux États-Unis entre 1980 et 1988}

HOULE (Martha), The fictions of casuistry and Pascal's Jesuit in Les Provinciales, University of California in San Diego, 1983, 297 p.

KELLY (Alton), Polemical language in Pascal's Pensées, University of North Carolina in Los Angeles, 1983, $338 \mathrm{p}$.

KOCH (Erec), Rhetoric and dialectic in Pascal, Yale University, 1988.

GALLUCCI (John), Discours humain et mystère divin : Pascal et l'attente de la vérité, Yale University, 1988.

LAGARDE (François), Pascal Christian : figures and reality of religion, Stanford University, 1985, 192 p. NESBITT (James), Discours polémique dans les Lettres Provinciales de Pascal, Middlebury College, 1983, $209 \mathrm{p}$.

STERN (Esther), Pascal and the Jews, City University of New York, 1981, 220 p.

TYMA (Deborah), Tagmemic grammar and reference in the Pensées of Pascal, University of Michigan, 1982, 486 p. 


\section{NOTES}

1. Hugh Davidson (Université de Virginie).

2. Anthony Pugh (Université de New Brunswick).

3. Robert Nelson (Université de l'Illinois).

4. Jan Miel (Université Wesleyan).

5. Lan Heller (Université du Western Ontario).

6. David Jaymes (Université de Rochester).

7. Buford Norman (Université de Caroline du Sud).

8. David Wetsel (Université de l'Arizona).

9. Charles Natoli (St John Fisher College, État de New-York).

10. Sara Melzer (Université de Californie, Los Angeles).

11. Louis MacKenzie (Université de Notre-Dame, Indiana).

12. Domna Stanton (Université du Michigan, Ann Arbor).

13. Barbara Woshinsky (Université de Miami).

14. Milad Doueihi (Université Johns Hopkins).

15. John Gallucci (Université Colgate, état de New-York).

\section{INDEX}

Mots-clés : Pascal

Index géographique : Amérique

\section{AUTEUR}

\section{PIERRE FORCE}

Columbia University 